\title{
Influence de la période nocturne sur la concentration plasmatique de 3-méthylhistidine chez la vache en production
}

\author{
BB Ndibualonji 1, D Dehareng 2, JM Godeau ${ }^{1}$
}

\begin{abstract}
'Laboratoire de Biochimie, Faculté de Médecine Vétérinaire, Université de Liège, Bd de Colonster, $n^{\circ} 20, B 42$, 4000 Liège-Sart Tilman, Belgique ; 2Faculté d'Agronomie, Université du Burundi, BP 2940, Bujumbura, Burundi
\end{abstract}

Dans une étude antérieure menée chez la vache tarie (Ndibualonji et al, Reprod Nutr Dev, in press), nous avons observé, d'une part, que la mobilisation protéique était plus importante pendant la sous-alimentation énergétique que pendant la sous-alimentation azotée, et, d'autre part, que les animaux mobilisaient les protéines labiles pendant la période nocturne pour satisfaire leurs besoins énergétiques.

Le but du présent essai est d'étudier les variations de la 3-méthylhistidine du plasma $(\mathrm{MeHi})$, index du catabolisme des protéines musculaires (Nagasawa et al, 1993, Biosci Biotech Biochem, 57, 517), pendant la période nocturne chez la vache en production. L'essai a été réalisé sur deux vaches adultes recevant deux repas journaliers égaux $(06.15 \mathrm{~h}$ et $15.30 \mathrm{~h}$ ) en fonction de leurs besoins d'entretien et de production. Les prélèvements de sang veineux ont été effectués toutes les 20 min à l'aide d'une seringue via un cathéter intrajugulaire de 06 à $14 \mathrm{~h}$ (période diurne) et de 22 à $06 \mathrm{~h}$ (période nocturne) à 1 semaine avant et à $1,4,8$ et 12 semaines après vêlage. Le dosage de la MeHi a été effectué par HPLC. Les données individuelles (diurnes ou nocturnes) ont été soumises à une analyse de la variance tandis que les différences entre les niveaux moyens enregistrés pendant la période diurne et ceux enregistrés pendant la période nocturne ont été déterminées grâce au test $t$. La comparaison des besoins des vaches aux apports alimentaires montre un déficit en protéines digestibles dans l'intestin (PDI) seulement en 1ère semaine de lactation tandis qu'un déficit énergétique se manifeste de la 1ère à la 8ème semaine de lactation.

Comparativement à la dernière semaine de gestation, la concentration en $\mathrm{MeHi}$ enregistrée pendant la période diurne augmente $(P<0,05)$ pendant la 1ère et la 4ème semaine de lactation, indiquant une importante mobilisation des réserves protéiques au cours des 4 premières semaines post-partum. Par ailleurs, quelle que soit la date du prélèvement sanguin, la concentration nocturne en $\mathrm{MeHi}$ a été plus élevée que la concentration diurne correspondante, la signification statistique $(P<0,05)$ n'étant cependant atteinte que pendant les 8 premières semaines de lactation où les besoins énergétiques des vaches n'étaient pas couverts, ce qui soutient nos résultats obtenus chez la vache tarie.

En conclusion, les résultats montrent que la vache, qu'elle soit en gestation ou en lactation, mobilise ses protéines endogènes pendant la période nocturne et que cette mobilisation est d'autant plus importante que les besoins énergétiques de production sont supérieurs aux apports alimentaires.

\begin{tabular}{lccccc}
\hline Semaines $\times$ & -1 & +1 & +4 & +8 & +12 \\
\hline Lait $(4 \% \mathrm{MG}, \mathrm{kg})$ & - & 15 & 17 & 18 & 16 \\
Poids vif $(\mathrm{kg})$ & 567 & 512 & 495 & 486 & 499 \\
PDI (\% besoins) & 129 & 98 & 107 & 104 & 118 \\
UFL (\% besoins) & 107 & 89 & 97 & 95 & 107 \\
MeHi $(\mu \mathrm{M})$ & & & & & \\
$\quad$ - diurne & $13^{\mathrm{a}}$ & $18^{\mathrm{b}}$ & $17^{\mathrm{b}}$ & $16^{\mathrm{ab}}$ & $14^{\mathrm{ab}}$ \\
$\quad$ - nocturne & $16^{\mathrm{a}}$ & $26^{\mathrm{b}^{*}}$ & $23^{\mathrm{b}^{*}}$ & $2 \mathrm{~b}^{*}$ & $17^{\mathrm{a}}$ \\
\hline
\end{tabular}

$\times$ Par rapport à la date du vêlage. a,b Les moyennes diurnes ou nocturnes sans lettre commune en indice sont différentes $(P<0,05)$. "valeur différente $(P<0,05)$ de celle enregistrée pendant la période diurne. 